ELUA

ISSN 2171-6692

Núm. 36, 2021, págs. 13-25

https://doi.org/10.14198/ELUA.2021.36.01

\title{
Reflexiones sobre la posesivización en español. Una aproximación cognitiva a las construcciones nominales con posesivo antepuesto
}

\author{
Perspectives on possessivization. A cognitive approach to the spanish nominal \\ constructions with a preceding possessive adjective
}

\author{
María Teresa Burguillo Escobar \\ Universidad Complutense de Madrid, España \\ maburgui@ucm.es \\ https://orcid.org/0000-0002-4001-797X
}

\begin{abstract}
Resumen
El objetivo del presente trabajo es proponer una nueva explicación de las construcciones nominales con posesivo antepuesto en español. Partimos de la revisión de las ideas defendidas en trabajos precedentes, muy especialmente en la Gramática Descriptiva de la Lengua Española y en los manuales que marcan la línea explicativa estándar, como los Cuadernos de Lengua
\end{abstract}

\begin{abstract}
The pivotal objective of this research is to propose a new explanation of a nominal construction with a preceding possessive adjective in the Spanish language. Therefore, the starting point is the review of the theoretical background of this concept in earlier works: the Gramática Descriptiva de la Lengua Española and the handbooks that mark the standard explanatory
\end{abstract}

Este trabajo se ha realizado gracias a la beca de Formación de Profesorado Universitario (FPU19/02293), del Ministerio de Educación y Formación Profesional, y al proyecto Diretes: Diccionario Reticular Español. Diccionario analógico y relacional con acceso en red desde el sentido y desde la forma" (FFI-2017-83293).

Agradezco a la profesora Silvia Iglesias Recuero su guía en la investigación que aquí presento y su generosidad intelectual en estos tiempos de exacerbada capitalización del conocimiento. En cada conversación, sus ideas han iluminado mi pensar. Gracias por tu sabiduría. Agradezco a la profesora María Auxiliadora Barrios Rodríguez y al proyecto Diretes (http://diretes.es/), del que es directora, el enriquecimiento en la reflexión, su espléndida cercanía en la colaboración, los acertados consejos a todos los niveles y la posibilidad de difusión del presente trabajo a través de esta publicación. Gracias por tu supervisión cautelosa y siempre amable.

Por último, gracias al Instituto Universitario Menéndez Pidal por su apoyo y al Ministerio de Universidades por la financiación de mis investigaciones a través de la ayuda FPU (FPU19/02293).

Para citar este artículo: Burguillo Escobar, M. T. (2021). Reflexiones sobre la posesivización en español. Una aproximación cognitiva a las construcciones nominales con posesivo antepuesto. ELUA, (36), 13-25. https://doi.org/10.14198/ELUA.2021.36.01

Recibido: 30/07/2021, Aceptado: 13/10/2021

(C) 2021 María Teresa Burguillo Escobar

Este trabajo está sujeto a una licencia de Reconocimiento 4.0 Internacional de

Creative Commons (CC BY 4.0) 
Española o la Enciclopedia de Lingüística Hispánica. En primer lugar, se analiza la concepción lingüística general que sustenta la explicación clásica del papel del posesivo en estas construcciones, se ofrece una compendiosa caracterización del fenómeno de la posesivización y se reflexiona acerca de los juicios de gramaticalidad que han sido propuestos como aval de las hipótesis tradicionales, así como sobre las condiciones para la posesivización enunciadas canónicamente. Tras una recopilación de los problemas de las explicaciones tradicionales, se pasa a ofrecer un giro de perspectiva a través de los principios de la lingüística cognitiva. Esencialmente, se utilizarán las herramientas teóricas de los marcos experienciales (Fillmore 1985), la prominencia ontológica y cognitiva (Anderson 1983: 86-125; Deane 1992: 34-35; Collins y Quillian 1969; Collins y Loftus 1975; Schmid 2007) y los puntos de referencia y el acceso mentalmente secuenciado a la referencia (Langacker 2009). A partir de este aparato de análisis y con la incorporación puntual y breve de la teoría de la metáfora (Lakoff y Johnson 1980), principalmente de la metáfora ontológica de objeto, se propone una alternativa explicativa a las construcciones nominales con posesivo antepuesto en español que, no solo incorpora como ejemplo central de la propuesta los casos de posesión stricto sensu, a saber, las relaciones de 'pertenencia o propiedad' (las cuales son desplazadas del centro explicativo en la mayoría de las obras canónicas), sino que se da cabida a aquellos contextos en los que los juicios de gramaticalidad son complejos y no evidentes en una primera lectura.

PALABRAS CLAVE: construcciones posesivas; posesión adnominal; posesivización; prominencia cognitiva; acceso mentalmente secuenciado. line, such as Cuadernos de Lengua Española and Enciclopedia de Lingüistica Hispáni$c a$. After the analysis of the general linguistic concept that supports the classical explanation of the role of the preceding possessive in the nominal constructions of the Spanish language, this study proposes a compendious characterization of the phenomenon of possessivization. Both grammatical judgements (proposed as endorsement of the traditional hypotheses) and the canonically enunciated conditions for possessivization are examined. A compilation of the problems regarding traditional explanations, and a perspective through the principles of Cognitive Linguistics is offered. Essentially, we resort to the theoretical tools of experiential frames (Fillmore 1985), ontological and cognitive prominence (Anderson 1983: 86125; Deane 1992: 34-35; Collins and Quillian 1969; Collins and Loftus 1975; Schmid 2007), as well as the reference points and mentally sequenced access to reference (Langacker 2009). Commencing from this analytical viewpoint we propose a new explanation of these constructions with the timely and brief incorporation of the theory of metaphor (Lakoff and Johnson 1980), mainly of the ontological metaphor. This new perspective incorporates as a central example the cases of possession stricto sensu, that is, the relationship of 'belonging or property' (which are displaced from the explanatory centre in most canonical works), and it also accommodates those contexts where the judgments of grammaticality are complex and not evident in the first reading.

KEY WORDS: possessive constructions; adnominal possession; Possessivization Principle; cognitive salience; sequenced mental access.

\section{INTRODUCCIÓN}

La noción de 'posesión' representa un reto para el lingüista que trate de ajustar la correspondencia entre forma y significado en el sistema de la lengua. Por una parte, existe una idea cotidiana de 'posesión' que depende de la cultura, de aspectos legales e históricos y de factores biológicos del ser humano. Por otra parte, es posible dar con una idea lingüística 
de 'posesión' asociada, bien con las construcciones que en una lengua proveen al hablante de la vía de expresión para ese concepto cotidiano de 'posesión' recién aludido, bien con todos los significados potenciales de dichas construcciones, que, por ser formalizados mediante construcciones posesivas, consideramos que tienen alguna relación semántica con la posesión o, de hecho, que significan 'posesión'.

En este sentido, indagando en las maneras de explicar la posesión, Heine (2001: 311) se plantea lo siguiente: “¿Hasta qué punto es posible estudiar las construcciones posesivas como un fenómeno exclusivamente lingüístico?".

En efecto, abordar la expresión lingüística de los significados posesivos precisa de una investigación transdisciplinar que acote desde un punto de vista antropológico, sociológico, histórico y psicológico, como mínimo, el significado posesivo que el lingüista analiza en las muestras de lengua.

Seiler (1983: 2-4), de hecho, define la posesión como un dominio "bio-cultural" que implica una relación entre dos entidades, un poseedor prototípicamente humano y que suele constituir informativamente un tópico, y un possessum, que habitualmente encierra el comentario. En esas relaciones entre el poseedor y lo poseído (o el possessum) caben diversos tipos: el vínculo entre un ser humano y sus parientes, iguales o personas de su entorno (kinsmen); entre un ser humano y las partes de su cuerpo; entre un ser humano y sus pertenencias materiales; entre un ser humano y sus productos culturales e intelectuales; $y$, desde un punto de vista amplio, entre las partes y el todo de un organismo. Añade Seiler que la posesión es una relación "estrictamente binaria", esto es, no hay poseedor sin poseído, y viceversa.

Teniendo en cuenta esto, nos acogemos a la distinción de Langacker (2009: 82), que propone desde una perspectiva cognitiva la caracterización semántica de la posesión basada en dos niveles: el nivel del prototipo (the prototype level) y el nivel del esquema (the schema level). El prototipo hace referencia al arquetipo conceptual surgido de la experiencia humana que caracteriza comúnmente la relación posesiva (esto es, el dominio "bio-cultural" de Seiler). Las relaciones prototípicas son, principalmente y desde el punto de vista de la tipología lingüística, la relación de pertenencia (habitualmente considerada como 'la posesión stricto sensu'), la de parte-todo y la de parentesco. En este trabajo, no nos centraremos en caracterizar estas relaciones y otras semánticamente vinculadas a la posesión, sino que discutiremos el nivel del esquema en el que se manifiestan las construcciones posesivas. Es en este nivel donde trabajan principalmente las explicaciones tradicionales y donde vamos a contraponer la visión clásica formal con la perspectiva cognitiva. Con el nivel del esquema nos referimos a un nivel abstraído de significado semántico-léxico, e instaurado en la forma o la instrucción lingüística como patrón inherente al sistema, según algunas visiones de la lengua, o inherente a la cognición y proyectado en el sistema, según otras visiones.

Partiendo, entonces, del nivel de esquema y desde una perspectiva onomasiológica, podemos distinguir las siguientes realizaciones lingüísticas de la posesión:

a) Posesión predicativa: Helena tienelposee una gata; La gata pertenece a Helena.

b) Posesión atributiva o posesión adnominal (o posesión interna): La gata de Helena; La gata suya; Su gata.

La diferencia principal entre la posesión predicativa y la atributiva es que, mientras que en la predicativa la relación posesiva se asevera explícitamente, en la atributiva está presupuesta o implícita. Se asocia, además, la posesión predicativa a la estructura oracional y la atributiva 
a la estructura del grupo nominal. Existe, también, un tipo de construcción intermedia, denominada posesión externa, del tipo Le atropellaron la gata, en la que la relación de posesión no aparece explicitada en el léxico del verbo, pero tampoco se ubica en el grupo nominal del elemento poseído: se ve representada a nivel oracional como un constituyente independiente.

El objetivo de este trabajo se centra en las construcciones posesivas atributivas o adnominales del tipo su gata y contrapone la explicación clásica formal de la aparición del posesivo en estas construcciones con el esbozo de una propuesta de perspectiva cognitiva que da cabida a los huecos que la explicación clásica deja vacíos.

La estructura del trabajo es la siguiente. En primer lugar, se expondrá el estado de la cuestión de las construcciones nominales con posesivo antepuesto en español mediante la revisión de la Nueva Gramática de la Lengua Española, la Gramática Descriptiva de la Lengua Española y la Enciclopedia de Lingüistica Hispánica, principalmente. A continuación, se ofrecerá una recopilación de los problemas existentes en las explicaciones canónicas de estas construcciones. Más adelante se propondrá una línea explicativa de las construcciones a partir de herramientas teóricas de corte cognitivista. Por último, se ofrecerán las conclusiones más relevantes del estudio.

\section{LAS CONSTRUCCIONES NOMINALES CON POSESIVO ANTEPUESTO EN ESPAÑOL: ESTADO DE LA CUESTIÓN}

Una característica esencial de los posesivos es que, al igual que los pronombres, contienen una referencia propia. Esta referencia es necesariamente distinta de la del sintagma nominal en el que esté inserto el posesivo. «Los posesivos son elementos deícticos, al igual que los pronombres personales» (RAE 2009: 1343). Esta característica ha llevado a que sean tratados en las obras clásicas como pronombres en genitivo independientemente de su posición en el sintagma nominal o sintagma determinante.

Partiendo de esta premisa, el estado actual de nuestras gramáticas descriptivas de la lengua española revela un tratamiento de las construcciones nominales con posesivo antepuesto (del tipo su carpeta) a partir de la asunción de la existencia de un indiscutible vínculo de carácter transformacional entre el posesivo antepuesto y el complemento del nombre al que, se entiende, "corresponde" el posesivo (la carpeta de Lola).

(1) a. $\left[\text { La carpeta de }[\text { Lola }]_{i}\right]_{j}>$ b. $\left[[\mathrm{Su}]_{\mathrm{i}} \text { carpeta }\right]_{\mathrm{j}}$

La correferencialidad (en que se basa tal correspondencia) entre Lola en la primera secuencia y el posesivo su en la segunda secuencia está marcada a través de la idéntica indexación de los elementos en (1). Por consiguiente, la transformación de (1) a. en (1) b. se asume intuitiva puesto que (1) b. se presenta como una paráfrasis aparentemente natural y respetuosa con las condiciones de vedad de (1) a.

Partiendo de esta consideración, el funcionamiento semántico de los posesivos antepuestos ha sido habitualmente explicado como idéntico a "las funciones semánticas de los argumentos del nombre que corresponden al caso genitivo, en particular, de los sintagmas nominales (...) introducidos por la preposición de" (Picallo y Rigau 1999: 980). Las siguientes secuencias traídas con fin ilustrativo proceden del mencionado texto de estas autoras, aunque su disposición y la notación referencial es propia: 
(2) a. $\left[\text { La tía de }[\text { Carlos }]_{\mathrm{i}}\right]_{\mathrm{j}}>\left[[\mathrm{Su}]_{\mathrm{i}} \text { tía }\right]_{\mathrm{j}}$

b. $\left[\mathrm{El} \text { ordenador de }[\mathrm{Gates}]_{\mathrm{i}}\right]_{\mathrm{j}}>\left[[\mathrm{Su}]_{\mathrm{i}} \text { ordenador }\right]_{\mathrm{j}}$

Eguren (2016: 867) propone que "la posición básica de los posesivos es la posnominal", de forma que "los posesivos antepuestos ocuparían una posición derivada debido a su carácter átono”. Si entendemos que la codificación semántica del posesivo antepuesto es la misma que la de los complementos del nombre a los que corresponden, entonces no solo la función semántica, sino, también, la relación semántica con el núcleo nominal ha de ser idéntica. $\mathrm{Y}$ es precisamente en este punto en el que los lingüistas encuentran el primer problema: la gama de relaciones entre el núcleo nominal y los complementos adnominales genitivos es muy amplia. Los ejemplos de (2) pueden ser continuados con una lista como la de (3):

(3) a. $\left[\text { La pata de }[\text { la mesa }]_{\mathrm{i}}\right]_{\mathrm{j}}>\left[[\mathrm{Su}]_{\mathrm{i}} \text { pata }\right]_{\mathrm{j}}$

b. $\left[\text { La foto de }[\text { Helena }]_{i}\right]_{j}>\left[[\mathrm{Su}]_{\mathrm{i}} \text { foto }\right]_{j}$

c. $\left[\text { El tren de }[\text { Pepe }]_{\mathrm{i}}\right]_{\mathrm{j}}>\left[[\mathrm{Su}]_{\mathrm{i}} \text { tren }\right]_{\mathrm{j}}$

En (3) queda de manifiesto que la relación que se establece entre el posesivo y el núcleo nominal no solo varía según la secuencia concreta, sino que ofrece también varias posibilidades interpretativas para una misma secuencia. Observemos a modo de ejemplo un breve análisis interpretativo de las secuencias recién expuestas. Por un lado, en (2) a. interpretamos una relación de 'parentesco' y, en (3) a., una relación de 'parte-todo'. Estas relaciones resultan evidentes a la intuición en una primera lectura. Sin embargo, en (3) c., la relación entre la entidad referida por Pepe y la entidad referida por tren puede ser de 'uso frecuente', de 'manejo habitual', de 'reciente adquisición', etc. Lo mismo sucede con (3) b., donde la entidad denotada por Helena puede ser 'el objeto representado en la foto', 'la propietaria de la foto', 'la persona que ha realizado la foto', etc. En (2) b. ocurre igual: Gates puede ser el propietario o el creador del ordenador, entre otras posibilidades. Este abanico semántico se extiende asimismo a la interpretación de (2) a. y a la de (3) a., que habíamos calificado de evidentes anteriormente: la tía de Carlos también puede aludir a aquella tía de Pepe, por ejemplo, de la que siempre habla Carlos, o a aquella tía del protagonista de la novela de Carlos, personaje del que últimamente no para de hablar, suponiendo que Carlos sea escritor; la pata de la mesa puede hacer referencia a aquella pata que acabo de comprar para restaurar la mesa y cambiar la que tiene rota, entre otras interpretaciones.

Para dar respuesta a esta aparente indefinición semántica, se ha convenido en explicar que la relación de posesión entre los referentes aludidos por el posesivo antepuesto y por el núcleo nominal es una relación de posesión laxa que se interpreta según el caso concreto (Picallo y Rigau 1999). A partir de aquí se establecen dos tipos de escenarios.

El primero de ellos corresponde a aquellos nombres que cuentan con estructura argumental, ya sea intrínseca (como los nombres representacionales, (4) a., o los nombres de parentesco, (4) b.) o heredada (nombres deverbales, (5) a., y deadjetivales, (5) b.). En ellos, la relación semántica entre el posesivo antepuesto y el núcleo nominal es la misma que la que establece el núcleo nominal en su estructura argumental con sus complementos seleccionados (el posesivo, de hecho, es considerado desde esta perspectiva tradicional un argumento del nombre):

(4) a. El retrato de Lola $>$ Su retrato

b. La nieta de María $>$ Su nieta 
(5) a. La exposición de Pepe > Su exposición

b. La prudencia de Lola $>$ Su prudencia

El segundo escenario está formado por aquellos nombres que no cuentan con estructura argumental (6), para los cuales los lingüistas han propuesto la existencia de una 'relación R' que actúa como nexo interpretativo entre el núcleo nominal y el complemento del nombre (que es susceptible de ser pronominalizado por el posesivo antepuesto) (Higginbotham 1983):

(6) La canción de Pepe $>$ Su canción

Esta 'relación R' es un “nexo semántico difícilmente definible en términos estrictamente léxicos", por lo que "suele interpretarse en muchos casos según el contexto discursivo o los conocimientos o intereses que comparten hablante y oyente" (Picallo y Rigau 1999: 980). No obstante, la 'relación R' no solo funcionaría para explicar el vínculo semántico entre posesivo y núcleo nominal en las estructuras con nombre sin selección argumental, sino que también aportaría la correcta elección interpretativa en los casos de nombres con estructura argumental del tipo de los ejemplos que comentábamos más arriba, (2) a., (3) a. y (3) b.

Con esto, las gramáticas descriptivas del español explican que los posesivos antepuestos pronominalizan aquellos complementos adnominales introducidos por la preposición de (Picallo y Rigau 1999) que tienen el estatus de argumento, porque están seleccionados por la semántica o la caracterización léxica del nombre (nombres con estructura argumental intrínseca o heredada). Si el núcleo nominal no cuenta con estructura argumental, se propone la existencia de una 'relación R' entre el núcleo nominal y el posesivo.

De esta manera, se entiende que la explicación excluye secuencias como las de (7) de las posibilidades ofrecidas por el sistema porque no cumplen con la condición propuesta por las explicaciones tradicionales para la pronominalización. Según Picallo y Rigau (1999: 984), "la imposibilidad de estas construcciones puede atribuirse al hecho de que el complemento adnominal ni es un verdadero argumento del núcleo, ni establece con este una 'relación R'”.

(7) a. Las películas de $\underline{\text { los domingos }}{ }_{\mathrm{i}}>*$ Sus $_{\mathrm{i}}$ películas

b. La taberna de la esquina ${ }_{\mathrm{i}}>* \mathrm{Su}_{\mathrm{i}}$ taberna

c. Las figurillas de terracota. ${ }_{i}>*$ Sus $_{\mathrm{i}}$ figurillas

Partiendo de estas líneas explicativas, han florecido trabajos que han continuado profundizando en la aparición del posesivo antepuesto en las construcciones nominales. Escandell (1999: 270) propone el término posesivización para este fenómeno de pronominalización de un complemento adnominal por un posesivo antepuesto, pues asume que "los posesivos átonos pueden reemplazar pronominalmente a los argumentos de los nombres". Establece, entonces, dos condiciones para la posesivización: la primera, que recoge lo expuesto en las gramáticas descriptivas, es la cualidad de 'argumento' que ha de poseer el complemento adnominal que va a ser posesivizado; la segunda, que constituye la aportación particular de su trabajo en el asunto, es que "solo los argumentos que el núcleo selecciona categorialmente como SSNN -y no los que selecciona como SSPP- pueden ser sustituidos por un posesivo" (1997: 60).

Para salvar de estas condiciones las posesivizaciones de complementos no argumentales, sino en 'relación R', la autora arguye que la 'relación R' convierte en argumentales tanto al 
núcleo nominal como al complemento/posesivo (no todos los lingüistas concluyen que la 'relación R' despliegue una selección argumental).

Por otra parte, y con respecto a los complementos adnominales procedentes de complementos de régimen, que sí son seleccionados como sintagmas preposicionales y no como sintagmas nominales, la autora defiende que las secuencias de nombres deverbales procedentes de verbos que requieren complemento de régimen resultan agramaticales si se posesiviza el complemento. El ejemplo a continuación y el juicio de gramaticalidad negativo proceden de Escandell (1999: 270).

(8) La dependencia del tabaco $\underline{i}_{i} . * \underline{\operatorname{Su}}_{\mathrm{i}}$ dependencia

Sin embargo, este juicio de gramaticalidad debería ser sometido a debate. Es posible aportar una lista de ejemplos donde la posesivización de complementos del nombre procedentes de complementos de régimen resulta perfectamente posible, como se muestra en (9).

(9) a. El abuso del alcohol $\underline{i}_{i}>\underline{\mathrm{Su}}_{\mathrm{i}}$ abuso $^{1}$

b. La carencia de hierro ${ }_{i} .>\underline{\mathrm{Su}}_{\mathrm{i}}$ carencia [RAE 2009: 1365]

c. La desposesión del inmueble $\underline{i}_{i}>\underline{\mathrm{Su}}_{\mathrm{i}}$ desposesión $^{2}$

\section{PROBLEMAS DE LAS EXPLICACIONES TRADICIONALES}

Los problemas que surgen inevitablemente de las explicaciones clásicas de las construcciones nominales con posesivo antepuesto son los siguientes.

- La relación posesiva prototípica, la relación de 'pertenencia o propiedad', está desplazada del corazón de la explicación. Esta se basa en la argumentalidad, rasgo que, precisamente, en la relación de 'propiedad o pertenencia' no se da.

- No se justifica por qué los ejemplos en los que el núcleo nominal no despliega estructura argumental de ningún tipo, pero sí son considerados gramaticales (véanse (1), (2) b., (3) c. y (6)) en el apartado 2), cuentan con 'relación R' y los que, en las mismas condiciones (su núcleo nominal no selecciona argumentos) son considerados agramaticales (véase (7) en el apartado 2), no poseen 'relación R'. Pues, es claro que existe un tipo de relación entre el núcleo y el complemento en todos los casos. ¿Por qué ese vínculo no se etiqueta como 'relación R'?

- En general, las explicaciones tradicionales demuestran que ante la amplitud de relaciones semánticas que participan de la estructura nominal con posesivo antepuesto y la dificultad de encontrar semánticamente un patrón común que explique las construcciones, se ha recurrido al desplazamiento hacia la explicación puramente formal a través de la argumentalidad y la selección sintáctica. Sin embargo, como tampoco desde este emplazamiento se reúnen las construc-

1 "El alcohol, su abuso y dependencia, son consistentemente uno de los problemas que más daño producen en la sociedad" en https://psicologia.isipedia.com/segundo/psicopatologia/psicopatologia-parte-1/13-alcoholismo (última consulta: 24/10/2021).

2 “(...) la demostración del derecho propietario del inmueble y su desposesión por los demandados (...)” en https://www.derechoteca.com/jurisprudencia/sala-civil-liquidadora-auto-supremo-no-4041-2013-del-29-de-agostode-2013/ (última consulta: 24/10/2021). 
ciones nominales posesivas en un patrón único, las explicaciones quedan sustentadas en la agramaticalidad de juicios cuanto menos susceptibles de debate. Nos referimos, particularmente, a la agramaticalidad aducida para los ejemplos de (7), la cual constituye la base para considerar la argumentalidad una herramienta explicativa en estas construcciones (solo los complementos adnominales argumentales pueden ser pronominalizados por un posesivo). Sin embargo, si se dota a los ejemplos de (7) de un contexto textual previo solo mínimamente enriquecido, las secuencias no solo dejan de ser agramaticales, sino que son perfectamente posibles en nuestra lengua (lo que demuestra que la prohibición no está en el sistema lingüístico):

(10) a. Los domingos $\underline{\underline{L}}_{\mathrm{i}}$ y $\underline{\underline{\text { sus }}}_{\mathrm{i}}$ típicas películas de la tarde ${ }^{3}$

b. La pintoresca esquina $\underline{\text { su }}_{j}$ conocida taberna

c. $\underline{\text { La terracota }}_{\mathrm{k}}$ y $\underline{\text { sus }}_{\mathrm{k}}$ habituales figurillas en la Antigua Grecia

\section{PROPUESTA EXPLICATIVA PARA LAS CONSTRUCCIONES NOMINALES CON POSESIVO ANTEPUESTO DESDE UN ENFOQUE COGNITIVO}

El giro que supone la perspectiva cognitiva en la investigación impulsa, para el tema que nos ocupa, un cambio de visión ante la realidad lingüística: en lugar de partir de la pregunta que de alguna manera subyace a las explicaciones tradicionales, a saber, ¿qué rasgos léxico-semánticos han de existir necesaria y suficientemente en los ítems lingüísticos para que tenga lugar el esquema nominal con posesivo antepuesto?, se plantea, ¿qué tarea cognitiva realizamos cuando interpretamos un esquema nominal con posesivo antepuesto? Esto es, en lugar de plantearse como objetivo desentrañar el sistema lingüístico partiendo de que sabemos cómo es el mundo externo, el foco se ubica en desentrañar la actividad mental de hacer significativo el mundo a través del sistema lingüístico y el sistema lingüístico a través del mundo, pues se entiende que nuestra estructuración conceptual está proyectada en nuestro sistema lingüístico, el cual es un producto de nuestras habilidades cognitivas.

Teniendo en cuenta este cambio de punto de vista, proponemos que la codificación semántica de las estructuras nominales con posesivo antepuesto es una instrucción cognitiva concreta que se define a través del acceso mentalmente secuenciado a la referencia, tal como muestran la Tabla 1 y la Ilustración 1:

3 Supongamos una conversación entre amigos que tienen niños muy pequeños, y una tarde, en el parque, con los niños de unos y otros jugando mientras ellos charlan, recuerdan su época de estudiantes y uno de ellos dice: "Igualito que antes, cuando quedábamos todos los miércoles para ir al cine". Sería perfectamente posible que algún otro dijera "Ay, aquellas benditas tardes y sus películas...". En un caso así, hay una experiencia común que se ha activado en el discurso precedente, lo que permite las secuencias que estamos estudiando. Pero no siempre es necesaria dicha activación previa. Se puede contar con un dato de experiencia que el hablante da por conocido. De hecho, hemos encontrado un ejemplo de uso que lo corrobora: "los bajonazos de los miércoles y sus películas gratis" (C.J. Torres, 2015, Siete tentáculos sin cabeza, ed. Rossetti, p. 53). En este caso se parte del dato (el autor de la novela lo da por conocido) de que en España el cine ofrece descuentos los miércoles. 


\begin{tabular}{|c|c|}
\hline Su & carpeta \\
\hline Entidad 1 & Entidad 2 \\
(E1) & $($ E2) \\
\hline
\end{tabular}

Tabla 1: Construcción nominal con posesivo antepuesto. Instrucción cognitiva

'Busque la referencia de E2 en el ámbito evocado por E1'

Ilustración 1: Propuesta de instrucción cognitiva para la construcción nominal con posesivo antepuesto.

La Entidad 1 (E1) es referencialmente la entidad del mundo conceptualizada como 'poseedor' y la Entidad 2 (E2) es la entidad conceptualizada como 'lo poseído'. En la construcción nominal con posesivo antepuesto evocamos la E2 a partir de la E1. En la instrucción cognitiva propuesta se presupone que E1 evoca un ámbito o marco. Esta presuposición se basa en el hecho de que "todas las unidades lingüísticas evocan un marco semántico" (Croft y Cruse 2008: 65).

Los marcos semánticos constituyen una herramienta teórica y explicativa del Modelo de la Comprensión, la alternativa de Fillmore (1985) a la categorización en semántica basada en relaciones de inclusión de clases. La Semántica de Marcos propone que los modelos semánticos que se basan en relaciones organizativas siguiendo patrones de relaciones inclusivas según los rasgos semánticos de las palabras no tienen un reflejo en las taxonomías que naturalmente realiza nuestro cerebro. Ese modelo, más bien, correspondería con las taxonomías artificiales de la computación. Nuestra mente, por el contrario, no utiliza rasgos suficientes y necesarios para manejar conceptos, sino que los conceptos se encuentran dispuestos en nuestro sistema cognitivo a través de marcos semánticos que engloban grupos de conceptos asociados mediante la experiencia.

En este sentido es en el que se entiende que E1 evoca un marco experiencial: en tanto que la materialización lingüística del concepto evoca un conjunto de entidades asociadas en la experiencia por el mero hecho de ser proferida la expresión lingüística. Esta evocación, por supuesto, no se produce explícitamente como material lingüístico, sino que se trata de la activación de esas entidades asociadas a E1 en la periferia de la conciencia de la información compartida entre hablante y oyente. La periferia de consciencia es la red de entidades que resultan accesibles a la atención de manera tácita, como consecuencia de haber seleccionado un concepto y de haberlo perfilado en calidad de foco de la atención. Esta periferia de conciencia se denomina ámbito o dominio de la atención (Chafe 1994).

En el caso de la construcción que nos ocupa, la construcción nominal con posesivo antepuesto, el ámbito de la atención constituye el dominio de búsqueda para el acceso a la referencia de E2. La referencia de E2 se descubre como aquella entidad que, cumpliendo con la definición estándar del concepto ('ser una carpeta', siguiendo el ejemplo (1)), se ubica en el ámbito/marco evocado por E1.

De esta manera, la explicación tradicional y la explicación cognitiva del fenómeno se contraponen en lo siguiente. La explicación tradicional subraya el vínculo entre el posesivo y los complementos del nombre, de lo que concluye que existe una pronominalización del primero sobre el segundo (además de un desplazamiento, en el caso de los posesivos antepuestos), y propone como condiciones necesarias la argumentalidad del complemento del nombre que se ha entendido que da origen al posesivo y la existencia de una 'relación $\mathrm{R}$ ', principalmente para los casos en los que los que el nombre no despliega estructura 
argumental. La explicación cognitiva no considera que el posesivo esté pronominalizando ningún complemento y, por el contrario, subraya su papel anafórico: el posesivo recupera la referencia de un concepto aludido previamente en el discurso. Aunque el enfoque cognitivo permitiría explicar los posesivos del español objeto del presente estudio, hasta donde sabemos no se ha ofrecido por el momento ninguna explicación satisfactoria. La pregunta es: ¿cómo recupera el posesivo la referencia previamente aludida?

Partiendo de esto, la propuesta de este estudio para responder a esta cuestión es que hay dos condiciones para la posesivización de ese referente previo: que entre el referente recuperado (E1, el posesivo) y el nuevo referente (E2, el núcleo nominal) exista una asociación dentro de un marco experiencial y que, como acabamos de apuntar, E1 esté activa en la memoria del hablante y del oyente porque haya sido previamente perfilada en el discurso.

Con esta explicación cognitiva, no solo los ejemplos de (10) sí tienen cabida a diferencia de lo que sucedía con la perspectiva clásica (7), sino que además se da una respuesta a por qué habían sido considerados agramaticales en las descripciones tradicionales. El aparato explicativo para esto último es el que mostramos a continuación.

Cuando llevamos a cabo juicios de gramaticalidad sobre secuencias lingüísticas hacemos un acto de introspección y nos preguntamos a nosotros mismos si son posibles tales secuencias. Al autopreguntarnos, el material que nuestro cerebro nos ofrece para respondernos no está instalado en ningún tipo de conciencia especial asociada a nuestra cualidad de nativos en tal o cual lengua, sino que se trata de un simple compendio más o menos ordenado del recuerdo de aquello que hemos escuchado desde que empezamos a tener contacto con nuestra propia lengua. A nuestro juicio, a partir de este recuerdo de secuencias de habla, nuestro razonamiento deduce ciertos patrones o líneas repetidas en la expresión y así, de un modo implícito, reconocemos las nuevas secuencias que se nos plantean como algo posible o imposible en nuestro sistema lingüístico: no a partir de una conciencia activa de la estructura de nuestra lengua, sino, más bien, a partir de una impresión producto del recuerdo de aquello que alguna vez hemos oído y de si aquello fue similar a lo que oímos ahora.

Admitiendo que esta es la actividad que llevamos a cabo cuando juzgamos la gramaticalidad de una secuencia, sucede que, como seres humanos con una visión sobre el mundo irremediablemente antropológica, no derramamos nuestra atención del mismo modo sobre todas las entidades del universo. De hecho, hay entidades ontológicamente más destacables para nuestra atención que otras. Por ejemplo, le damos una mayor importancia a los agentes y a los seres animados, y una menor importancia a los objetos o entidades inanimadas e inmateriales. En lingüística cognitiva, esta cualidad de ciertas entidades se denomina prominencia ontológica (Schmid 2007) y es una propiedad más o menos estable de las entidades mismas. Sin embargo, la prominencia ontológica está sujeta a nuestra conceptualización de la realidad, así como al tipo de discurso en el que nos encontremos y a la frecuencia con la que las entidades han sido perfiladas como conceptos en calidad de foco de atención en los discursos. Este otro fenómeno se denomina prominencia cognitiva (Anderson 1983: 86-125; Deane 1992: 34-35; Collins y Quillian 1969; Collins y Loftus 1975; Schmid 2007).

Los ejemplos de (7) fueron juzgados como agramaticales en las explicaciones tradicionales porque, en contraste con los de (1), (2), (3) b., (3) c., (4), (5) y (6), en los que los poseedores son animados y humanos, los propuestos para (7) son entidades inanimadas, abstractas e incluso sin límites claros (como la 'realidad temporal' del domingo o la indefinición espacial de una esquina). Estas entidades propuestas para poseedores no tienen una alta destacabilidad desde 
un punto de vista ontológico, esto es, son poco prominentes ontológicamente. Además, no se ofrece ningún discurso previo en el que hayan sido perfiladas con anterioridad, con lo cual es muy costoso a nivel cognitivo recuperarlas de manera activa: supone un esfuerzo mayor imaginar un contexto donde puedan ser prominentes cognitivamente para que la posesivización pueda suceder sin problemas. (Para consultar escalas de prominencia, véanse Langacker (1991: 171 y 307), Schmid (2007: 133), Cifuentes (2015: 13)).

Estos factores de los que carece la enunciación de los ejemplos en (7) son con los que sí cuentan los ejemplos en (10). En ellos, las entidades propuestas para poseedores han sido todas activadas previamente en el primer elemento de la cópula, de manera que no resulta costoso recuperarlas anafóricamente porque ya están activas en el discurso, es decir, porque son prominentes cognitivamente. Su prominencia ontológica sigue siendo baja, porque las entidades tienen las mismas características que enunciamos para (7), pero en (10) han sido delimitadas (como se observa con el sustantivo de masa terracota $>$ la terracota). Esta delimitación entronca con la teoría de la metáfora y con las metáforas ontológicas de objeto (Lakoff y Johnson 1980).

En efecto, en Metaphors We Live By, los autores explican que uno de los instintos humanos más básicos es la 'territorialidad'. Una de las consecuencias de este instinto consiste en que imponemos límites (conceptuales) incluso a aquello que por naturaleza no lo tiene. Esto sucede, por ejemplo, con la realidad temporal (inaprensible), que es categorizada lingüísticamente a través del léxico espacial (aprensible) con el fin de acotarla para poder referirnos a ella. Así, mediante nuestro sistema cognitivo y lingüístico convertimos en 'objetos' las realidades abstractas ('el tiempo'> los domingos), las realidades físicas sin límites intrínsecos ('el espacio' > la esquina); delimitamos los nombres de masa ('terracota' > la terracota), etc. Esta actividad de metaforizar como objetos delimitados aquello que no posee fronteras precisas no es baladí: solo porque hacemos discreto conceptualmente lo que no lo es de modo natural, podemos razonar sobre ello, extraer aspectos, buscar causas, etc. Las entidades poseedoras en (10) han sido metaforizadas objetualmente y activadas previamente en el discurso, de manera que su prominencia general resulta mayor que en (7). Como consecuencia, los ejemplos de (10) resultan gramaticales, mientras que los de (7) han sido calificados de agramaticales.

Con esto, entendemos que no hay nada en el sistema que prohíba las secuencias de (7), por lo que concluimos que, en realidad, son gramaticales. El hecho de que se hayan emitido juicios de gramaticalidad negativos sobre (7) queda explicado por la falta de prominencia cognitiva de las entidades poseedoras en tales ejemplos.

\section{RECAPITULACIÓN Y CONCLUSIONES}

En este trabajo se ha discutido el estado de la cuestión de las construcciones nominales con posesivo antepuesto en español. Los trabajos clásicos, la NGLE, la Gramática Descriptiva de la Lengua Española, la Enciclopedia de Lingüistica Hispánica y Escandell (1999), tratan los posesivos antepuestos como pronombres en genitivo movidos a la posición de determinantes. Este tratamiento conlleva que los autores vinculen el contenido semántico de los posesivos al de los complementos adnominales a los que parece que pueden sustituir. Movidos por el hecho de que en muchas ocasiones estos complementos son argumentales, proponen una restricción sobre la aparición del posesivo según si este corresponde a un 
complemento adnominal que es o no argumento del núcleo nominal. Los inevitables huecos de esta explicación surgen al introducir casos prototípicos de la posesión como la relación de pertenencia o propiedad, donde no existe despliegue argumental por parte del núcleo nominal. Para dar respuesta a este problema, los autores han propuesto una solución ad hoc: estos casos cuentan con una 'relación R'. Sin embargo, la 'relación R' queda tan vagamente dibujada, que no se explica por qué los casos considerados como agramaticales por ser el complemento susceptible de pronominalización por un posesivo adjunto y no argumental no cuentan con 'relación R'.

La perspectiva cognitiva otorga la posibilidad de replantear la agramaticalidad de las secuencias consideradas como tales en las obras al respecto de corte más clásico al introducir dos herramientas teóricas muy útiles: los conceptos de 'prominencia ontológica' y 'prominencia cognitiva'. Las secuencias consideradas agramaticales a causa de la carencia de argumentalidad en el posesivo resultan totalmente gramaticales si la entidad a la que alude el posesivo está activada previamente en el discurso. Por este motivo, se observa que el vínculo entre el posesivo y el supuesto complemento del nombre al que las teorías tradicionales entienden que posesiviza no es de tal naturaleza transformacional o derivacional. La dirección es la opuesta: el posesivo engancha con una referencia previa y es por eso que la restricción fundamental para la aparición del posesivo antepuesto en construcciones nominales es que la referencia que recupera anafóricamente se encuentre activa para el hablante y para el oyente en el acto comunicativo.

Por consiguiente, no se trata de si el posesivo es un argumento o no del núcleo nominal, sino, simplemente de si se encuentran asociadas las entidades conceptualizadas como poseedor y como poseído en un marco experiencial, de manera que la activación del poseedor pueda conducir al acceso mentalmente secuenciado a la referencia de lo poseído.

Las futuras líneas de investigación que plantea este trabajo están relacionadas con el establecimiento de escalas de prominencia ontológica y cognitiva precisas (según el tipo de discurso) y la profundización en la naturaleza de los marcos experienciales que sirven de fuente para el acceso mentalmente secuenciado en las construcciones posesivas.

\section{REFERENCIAS BIBLIOGRÁFICAS}

Anderson, J. R. (1983). The architecture of cognition. Cambridgem MA: Harvard University Press.

Chafe, W. (1994). Discourse, consciousness and time: the flow and displacement of conscious experience in speaking and writing. Chicago: University of Chicago Press.

Cifuentes Honrubia, J. L. (2015). Construcciones posesivas en español. Leiden/Boston: Brill-Rodopi.

Collins, A. M. y E. Loftus (1975). "A spreading-activation theory of semantic processing", Psychological Review, 82, pp. 407-428.

Collins, A. M. y M. R. Quillian (1969). "Retrieval time for semantic memory", Journal of Verbal Learning and Verbal Behavior, 8, pp. 240-247.

Croft, W. y D. A. Cruse (2008). Lingüistica cognitiva. (A. Benítez Burraco, Trad.). Madrid: Akal. (Obra original publicada en 2004).

Deane, P. D. (1992). Grammar in mind and brain: Explorations in cognitive syntax. Berlin: Mouton de Gruyter.

Eguren Gutiérrez, L. (2016). "Pronombres personales”. En: Gutiérrez-Rexach, J. (coord.). Enciclopedia de Lingüística Hispánica, Vol. 1. Reino Unido: Routledge, pp. 859-869.

Escandell Vidal, M. V. (1997). Los complementos del nombre. Madrid: Arco Libros. 
Escandell Vidal, M. V. (1999). "Notas sobre la gramática de los posesivos”. En Gómez Manzano, P., Carbonero P. y M. Casado (eds.). Lengua y discurso: Estudios dedicados al profesor Vidal Lamíquiz. Madrid: Arco Libros, pp. 265-278.

Fillmore, C. J. (1985). "Frames and the semantics of understanding", Quaderni di semántica, 6, pp. 222-254.

Heine, B. (2001). "Ways of explaining possession”. En: Baron, I., Herslund, M. y F. Sørensen (eds.). Dimensions of possession. Ámsterdam/Filadelfia: John Benjamins Publishing Company, pp. 311328.

Higginbotham, J. (1983). "Logical Form, Binding and Nominals", LI, 14, pp. 395-420.

Lakoff, G. y M. Johnson (1980). Metaphors We Live By. Chicago: The University of Chicago Press.

Langacker, R. W. (1991). Foundations of Cognitive Grammar, Vol. 1 Theoretical Prerequisites. Stanford, California: Stanford University Press.

Langacker, R. W. (2009). Investigations in Cognitive Grammar. Berlín: Mouton de Gruyter.

Picallo i Soler, M. C., y G. Rigau i Oliver (1999). "El posesivo y las relaciones posesivas". En Bosque, I. y V. Demonte (eds.). Gramática Descriptiva de la Lengua Española. Madrid: Espasa, pp. 973-1023.

Real Academia Española (2009). Nueva gramática de la lengua española. Madrid: Espasa.

Schmid, H.-J. (2007). "Entrenchment, salience, and basic levels". En Geeraerts, D. y H. Cuyckens (eds.). The Oxford Handbook of Cognitive Linguistics. Oxford: Oxford University Press, pp. 117138.

Seiler, H. (1983). Possession as an operational dimension of language. Tübingen: Gunter Narr. 